\title{
Fluorescence induction of photosynthetic bacteria
}

\author{
G. SIPKA ${ }^{* * * *}$, M. KIS ${ }^{*}$, J.L. SMART ${ }^{* * *}$, and P. MARÓTI ${ }^{*+}$ \\ Department of Medical Physics, University of Szeged, Rerrich Béla tér 1, Szeged 6720, Hungary* \\ Department of Plant Biology, Hungarian Academy of Science, Biological Research Centre, Szeged, Hungary* \\ Department of Biological Sciences, University of Tennessee at Martin, USA ${ }^{* * *}$
}

\begin{abstract}
The kinetics of bacteriochlorophyll fluorescence in intact cells of the purple nonsulfur bacterium Rhodobacter sphaeroides were measured under continuous and pulsed actinic laser diode ( $808 \mathrm{~nm}$ wavelength and maximum $2 \mathrm{~W}$ light power) illumination on the micro- and millisecond timescale. The fluorescence induction curve was interpreted in terms of a combination of photochemical and triplet fluorescence quenchers and was demonstrated to be a reflection of redox changes and electron carrier dynamics. By adjustment of the conditions of single and multiple turnovers of the reaction center, we obtained $11 \mathrm{~ms}^{-1}$ and $120 \mu \mathrm{s}^{-1}$ for the rate constants of cytochrome $c_{2}{ }^{3+}$ detachment and cyclic electron flow, respectively. The effects of cytochrome $c_{2}$ deletion and chemical treatments of the bacteria and the advantages of the fluorescence induction study on the operation of the electron transport chain in vivo were discussed.
\end{abstract}

Additional keywords: bacterial photosynthesis; electron transfer; fluorescence transients; intact cells.

\section{Introduction}

The science-based celebration of Govindjee's birthday should be inevitably connected to the Kautzky effect of characteristic kinetic changes of the chlorophyll (Chl) fluorescence of photosynthetic organisms upon light excitation (Kautsky and Hirsch 1931). First, he was born a year after the effect was discovered, and second, he has shown life-long commitment to the phenomenon of Chl fluorescence induction while fascinating and motivating two or three generations of plant scientists. Largely thanks to his pioneering and seminal works on the interpretation and evaluation of the Kautzky effect, there has been a long tradition to track the cyclic and noncyclic electron transfer in higher plants, cyanobacteria, and algae by kinetic changes of the Chl fluorescence (Stirbet and Govindjee 2012). However, considerably fewer investigations and much lower level of instrumentation have been devoted to the bacteriochlorophyll (BChl) fluorescence of photosynthetic bacteria. In an attempt to reduce this gap, and in honor of Govindjee, the subject of this work is devoted to the induction and relaxation of $\mathrm{BChl}$ fluorescence in the purple nonsulfur photosynthetic bacterium Rhodobacter sphaeroides under various time and excitation ranges.

The topography of the photosynthetic apparatus of purple nonsulfur bacteria and the function of the macromolecular complexes including the intra- and interprotein electron transfer have been revealed with high precision (Niederman 2016). The system is set into operation by absorption of light in the light-harvesting complex. The electron excitation energy is funneled to the reaction center $(\mathrm{RC})$ where an electron is stripped from $\mathrm{BChl}$ dimer $(\mathrm{P})$ thereby acquiring a positive charge $\left(\mathrm{P}^{+}\right)$ (Fig. 1). The electron from the periplasmic site of the membrane is transferred via several different pigments to the acceptor quinone system $\left(\mathrm{Q}_{\mathrm{A}} \mathrm{Q}_{\mathrm{B}}\right)$ on the cytosolic membrane face thus producing a charge separation $\mathrm{P}^{+}\left(\mathrm{Q}_{\mathrm{A}} \mathrm{Q}_{\mathrm{B}}\right)^{-}$in the $\mathrm{RC}$. After accepting two electrons from the same $P$, the quinone binds two protons from the cytosol, forming the reduced quinone $\mathrm{QH}_{2}$, which is then released as a two-electron carrier from the $\mathrm{RC}$. The flow of electrons from the lipid-soluble $\mathrm{QH}_{2}$ to other acceptors proceeds via either a cyclic or noncyclic path. Following the cyclic pathway, the $\mathrm{QH}_{2}$ diffuses to a cytochrome $b c_{1}$

Received 16 May 2017, accepted 28 August 2017, published as online-first 26 September 2017.

${ }^{+}$Corresponding author; phone: 36-62-544-120, e-mail: pmaroti@sol.cc.u-szeged.hu

Abbreviations: $\mathrm{BChl}$ - bacteriochlorophyll; $\mathrm{Chl}$ - chlorophyll; $\mathrm{F}_{0}-$ minimal fluorescence yield of the dark-adapted state; $\mathrm{F}_{\max }-$ maximal fluorescence yield of the light-adapted state; $F_{v}$ - variable fluorescence.

Acknowledgements: We are indebted to Dr. Emese Asztalos for her contribution to the early phase of this work. The authors gratefully acknowledge financial support from GINOP-2.3.2-15-2016-00001, OTKA-K 112688, Photosynthesis - Life from Light - Foundation (Hungary) (G. Sipka), COST (CM1306), EFOP-3.6.2-16-2017-00001 (M. Kis and P. Maróti), the Bill and Roberta Blankenship CENS Research Endowment, the Hal and Alma Reagan Faculty Leave, and the van Dyck Faculty Leave (J.L. Smart). 


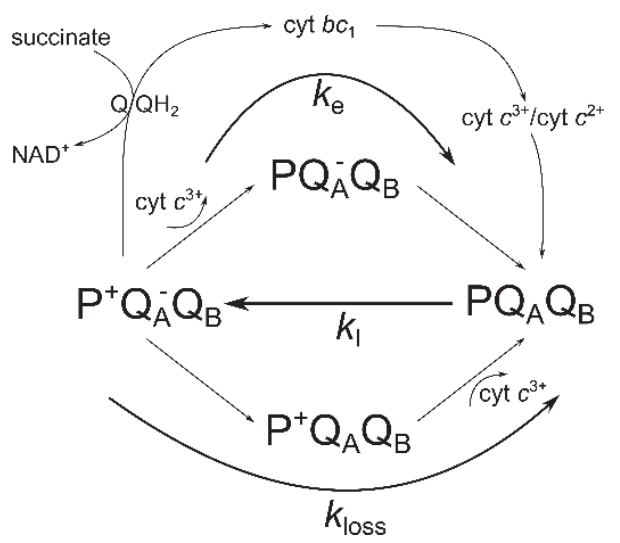

Fig. 1. A simplified scheme for stationary electron flows in and around the RC: light excitation closes the open $\mathrm{RC}\left(\mathrm{PQ}_{\mathrm{A}} \mathrm{Q}_{\mathrm{B}}\right)$ with rate constant $k_{\mathrm{I}}$ and the closed $\mathrm{RC}\left(\mathrm{P}^{+} \mathrm{Q}_{\mathrm{A}}^{-}\right)$is re-opened by electron transfer from the acceptor side, via the $\mathrm{Q} / \mathrm{QH}_{2}$ quinone pool in the membrane, the cytochrome $b c_{1}$ complex and the periplasmic cyt $\mathrm{c}$, arriving at the donor side, $\mathrm{P}$ with overall rate constant $k_{\mathrm{e}}$ (cyclic electron transport). The noncyclic (reverse) electron flow from succinate to $\mathrm{NAD}^{+}$is connected to the cyclic flow at the $\mathrm{Q} / \mathrm{QH}_{2}$ quinone pool in the membrane. If any of the positive or negative charges cannot escape from the RC (predominantly at single turnover of the $\mathrm{RC}$ ), the closed $\mathrm{RC}$ will be re-opened by a slow leakage of rate constant $k_{\text {loss }}$.

complex where it releases its two electrons to a site on the exoplasmic surface. Simultaneously, $\mathrm{QH}_{2}$ releases two protons into the periplasmic space, thereby generating a proton-motive force which is used by the $\mathrm{F}_{0} \mathrm{~F}_{1} \mathrm{ATPase}$ to synthesize ATP and also to transport molecules across the membrane. Electrons are then transferred from the cytochrome $b c_{1}$ complex to a water-soluble cytochrome (cyt $c_{2}$ ), a one-electron carrier in the periplasmic space; this reduces the cytochrome from an $\mathrm{Fe}^{3+}$ to an $\mathrm{Fe}^{2+}$ state. The reduced cytochrome then diffuses through a small pool of cyt $c_{2}{ }^{3+} /$ cyt $c_{2}{ }^{2+}$ back to the RC, where it releases its electron to the positively charged dimer $\left(\mathrm{P}^{+}\right)$, returning it to the ground state $\mathrm{P}$. In the case of the noncyclic pattern of reverse electron flow in purple nonsulfur bacteria, the electrons are removed from reduced organic molecules,

\section{Materials and methods}

Bacterial strains, culture conditions and chemicals: Purple nonsulfur photosynthetic bacterium $R$. sphaeroides wild type (WT) was grown in Siström's medium (Siström 1962) in completely filled screw top vessels without oxygen (anaerobic growth) under irradiance of $13 \mathrm{~W} \mathrm{~m}^{-2}$ from tungsten lamps (Asztalos et al. 2010). The cells were harvested at an exponential phase of the growth at cell concentration of $\sim 10^{8}$ cell $\mathrm{mL}^{-1}$ and were bubbled with nitrogen for $15 \mathrm{~min}$ before measurements to preserve the anoxic conditions.

Strain JS2293 $\Delta$, containing an in-frame deletion of the $c y c A$ gene encoding cytochrome $c_{2}$ in $R$. sphaeroides, was genetically constructed essentially as described previously such as succinate and lactate, and are transferred to $\mathrm{NAD}^{+}$ via the quinone pool in the membrane, forming NADH. In the noncyclic pathway, as in the cyclic pathway, a protonmotive force is generated and used to synthesize ATP.

In order to track the electron transfer steps and for the evaluation of the physiological status of the purple anoxygenic phototrophs, induction and relaxation of BChl fluorescence have largely prevailed (Koblizek et al. 2005, Maróti 2008, 2016; Asztalos et al. 2010, 2015; Kis et al. 2014, 2015). While the RCs are photooxidized and effectively become closed during the fluorescence induction phase, they open again during the fluorescence relaxation phase. The classic relation between the fluorescence yield and the fraction of closed RC has served as the basis for interpretation of fluorescence induction (Duysens 1951). When the $\mathrm{RC}$ is open $\left(\mathrm{PQ}_{\mathrm{A}}\right)$, the fluorescence level is low $\left(\mathrm{F}_{0}\right)$ as part of the excitation energy is used for photochemistry (charge separation). However, when the $\mathrm{RC}$ is closed $\left(\mathrm{P}^{+} \mathrm{Q}_{\mathrm{A}}^{-}\right)$, the level of fluorescence is high $\left(\mathrm{F}_{\max }\right)$ because photochemical loss no longer comprises the nonradiative dissipative pathway of the excited BChl. The difference between the maximum and minimum fluorescence (variable fluorescence $F_{v}=F_{\max }-F_{0}$ ) reflects the redox status of the $\mathrm{RC}$, and provides an estimate of the quantum yield of the primary charge separation (Trissl 1996, de Rivoyre et al. 2010).

In the present study, the fluorescence induction of the $\mathrm{RC}$ from $R$. sphaeroides is evaluated under high light (continuous) and moderate (intermittent) light intensities. The observed kinetic phases are the result of different types of fluorescence quenchers including photochemical quenchers (the oxidized quinones and the reduced dimer in the RC) and triplets of the excited states of $\mathrm{BChl}$ and carotenoids, and the ground state of oxygen. Under sufficiently low-light conditions, where the light-driven processes (charge separation and triplet formation) compete with electron transfer, a simple method is described to determine the overall steady-state rate of cyclic electron transfer in intact cells of $R$. sphaeroides. The effects of different electron transfer inhibitors and treatments of the bacteria are also discussed.

(Chi et al. 2015). Escherichia coli strains were grown at $37^{\circ} \mathrm{C}$ in LB medium (Sambrook et al. 1989) supplemented with antibiotics when appropriate; kanamycin $\left(50 \mu \mathrm{g} \mathrm{mL}^{-1}\right)$ and ampicillin $\left(100 \mu \mathrm{g} \mathrm{mL}^{-1}\right)$. R. sphaeroides strains were grown aerobically at $30^{\circ} \mathrm{C}$ in YCC medium (Siström 1977) supplemented when appropriate with kanamycin $(50 \mu \mathrm{g}$ $\left.\mathrm{mL}^{-1}\right)$. Conjugal transfer of strains from E. coli to $R$. sphaeroides was performed as described previously, and counter-selection against S17-1 donors was achieved by addition of tellurite $\left(100 \mu \mathrm{g} \mathrm{mL}^{-1}\right.$ ) (Donohue and Kaplan 1991).

Terbutryne was used in $120 \mu \mathrm{M}$ concentration to block the $\mathrm{Q}_{\mathrm{A}} \rightarrow \mathrm{Q}_{\mathrm{B}}$ interquinone electron transfer in the $\mathrm{RC}$ as 
terbutryne competes with quinone for the $\mathrm{Q}_{\mathrm{B}}$-binding site. Myxothiazol was used in $10 \mu \mathrm{M}$ concentration to inhibit the cyclic electron transfer because it is a competitive inhibitor of ubiquinol and binds at the quinol oxidation $\left(Q_{0}\right)$ site of the cytochrome $b c_{1}$ complex. The uncoupling agent, $p$-triflouromethoxy carbonilcyanide phenylhydrazone (FCCP), was applied in $20 \mu \mathrm{M}$ concentration to abolish the linkage between the respiratory chain and the phosphorylation system by dissipating the proton-motive force across the membrane.

Induction and relaxation of $\mathbf{B C h l}$ fluorescence: The induction and relaxation kinetics of the BChl $a$ fluorescence of intact cells were measured by a home-built fluorometer (Kocsis et al. 2010). The light source for both excitation and monitoring was a laser diode (wavelength of $808 \mathrm{~nm}$ and maximum light power of $2 \mathrm{~W}$ ) that

\section{Results}

Fluorescence induction under high light excitation: Fluorescence induction from the dark state, $\mathrm{F}_{0}$, to the maximum $F_{\max }$ within a couple of $\mu$ s (Fig. 2) occurred because of the high light intensity $\left(2 \mathrm{~W} \mathrm{~cm} \mathrm{~cm}^{-2}\right)$ and wavelength $(808 \mathrm{~nm})$, which matches the absorption band of the peripheral light-harvesting antenna of $R$. sphaeroides. After an additional small rise, it reached $\mathrm{F}_{\max }$ approximately $1 \mathrm{~ms}$ after onset of the excitation. From that level, the fluorescence drops slowly and continuously in several phases to approximately half of the maximal value. A small increase can be seen at the very end of the excitation in the vicinity of the min time range. The kinetic trace is reproducible and reflects reversible changes in the bacterium. This is in contrast to the behavior of free BChl in solution, and the carotenoid-less mutant R-26, where the bleaching of the pigments can be seen in the ms time range under similar induction conditions. It is evident from these experiments that the carotenoids protect the $\mathrm{BChl}$ and the photosynthetic apparatus effectively from the oxidative stress caused by the high light intensity. Upon termination of the light excitation, an increase and subsequent decrease of the fluorescence level can be observed at onset of the excitation after dark periods of different durations (data not shown). These are clear signs of relaxation of triplets in the dark and building up in the light. Although the triplets obviously determine the fluorescence kinetics, the contribution of other effects cannot be disclosed in the longer time scale. The definite increase of the fluorescence due to addition of myxothiazol (blocker of the cyt $b c_{1}$ complex) and a slight change upon addition of FCCP (uncoupler of electron and proton transfers) demonstrated the effects of cyclic electron transfer and transmembrane $\mathrm{pH}$ gradient on the $\mathrm{BChl}$ fluorescence, respectively.

Fluorescence induction upon intermittent excitation: The intensity of the excitation can be decreased by intermittent pulsing of the emission of the laser diode. The produced rectangular shape of illumination of variable duration and intensity and matched the $800-\mathrm{nm}$ absorption band of the $\mathrm{LH} 2$ peripheral antenna of the cells. The BChl fluorescence (centered at $900 \mathrm{~nm}$ ) was detected in the direction perpendicular to the actinic light beam with a near-infrared-sensitive, large-area (diameter $10 \mathrm{~mm}$ ), and high-gain Si-avalanche photodiode (APD; model 394-7072-581; Advanced Photonix, Inc., USA) protected from the scattered light of the laser by an $850-\mathrm{nm}$ high-pass filter $(R G-850)$. Solutions of BChl or IR-806 dye (Sigma) were used as reference for fluorescence yield measurements to correct for any deviations from the rectangular shape and for large-scale fluctuation of the laser diode excitation. The reference signal was adjusted to the same intensity as that of the fluorescence to avoid the possible artifact coming from the nonlinearity of the response of the detector at high light intensity.

bacteria were illuminated by a series of light flashes of $3-\mu$ s duration and the attenuation of the light intensity was controlled by changing the duty cycle (Fig. 3). The steadystate levels of the fluorescence decreased continuously with a decrease in the duty cycle and showed distinct fast and slow kinetic phases (Fig. $3 A$ ). At higher duty cycles, single turnover of the RC occurred causing fast decay of the fluorescence level from $F_{\max }$. This is probably due to fast reduction of $\mathrm{P}^{+}$by cyt $c_{2}{ }^{2+}$ bound or closely attached to the RC of $R$. sphaeroides. The slow component reflects the competition of the photochemical charge separation with the stationary flow of the electrons from and in the $\mathrm{RC}$ (multiple turnover of the RC). The observation of

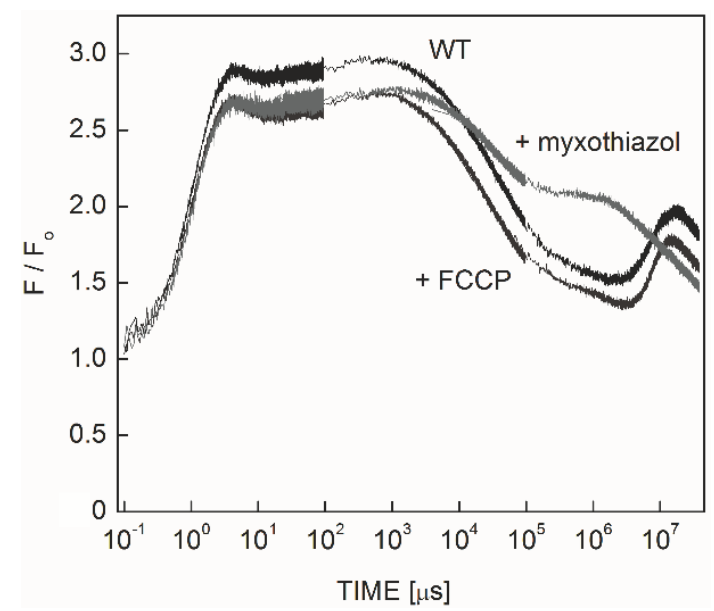

Fig. 2. Fluorescence induction $\left(\mathrm{F} / \mathrm{F}_{0}\right)$ of anaerobic intact and inhibitor (FCCP and myxothiazol)-treated cells of Rhodobacter sphaeroides upon strong and rectangular shape of excitation (intensity $2 \mathrm{~W} \mathrm{~cm}^{-2}$ at $808 \mathrm{~nm}$ ). $\mathrm{F}_{0}$ denotes the initial level of fluorescence. Note the logarithmic scales. Each trace was constructed by joining data sets from measurements of three separate time-ranges; these are visible as noise changes in the data. 


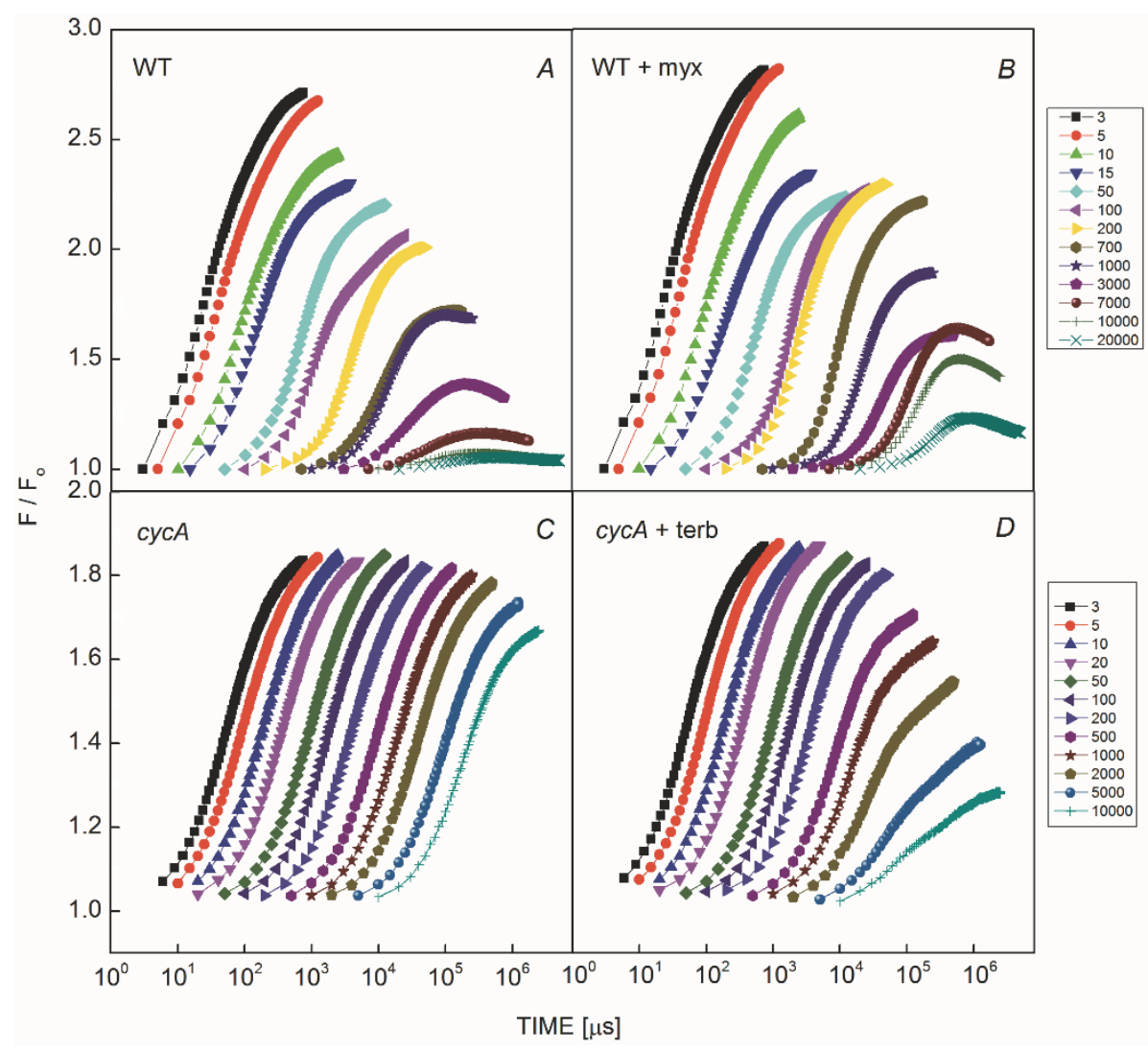

Fig. 3. Set of fluorescence induction curves of wild type $(A$ und $B)$ and cytochrome-less $(C$ and $D)$ cells of Rhodobacter sphaeroides without $(A$ and $C$ ) and with addition of $20 \mu \mathrm{M}$ myxothiazol $(B)$ or $120 \mu \mathrm{M}$ terbutryne $(D)$. The fluorescence was evoked by 250 flashes of 3- $\mu$ s duration with different dark periods between the neighboring flashes. By changing the duty cycle of the flash series (the ratio of pulse width to pulse period), the fluorescence induction at different light excitations can be measured (see the reciprocals of the duty cycle attached to the color codes). The photochemical rate constant $k_{\mathrm{I}}$ at arbitrary light intensity is determined from the measured value at the highest duty cycle multiplied by the duty cycle of the flash series.

slow decrease of the fluorescence level in the ms time range may reveal essential characteristics of the electron transfer around the RC. Inhibition of electron transfer to the auxiliary oxidants by the cyt $b c_{1}$ inhibitor myxothiazol can partly block the slow component without any effects on the initial charge export at the donor and shifts the decay of the fluorescence to larger time scale of seconds (Fig. $3 B$ ). If the export of the positive charge on the donor side is inhibited, no decrease of the fluorescence is expected anymore. Indeed, flat $\mathrm{F}_{\max }$ level and only a small subsequent drop in the 10 -s time range characterize the mutant in which the $c y c A$ gene is deleted (Fig. $3 C$ ). A somewhat faster decrease of the fluorescence level can be observed in the time scale of seconds if the electron transfer was inhibited by addition of terbutryne to the $c y c A$ mutant (Fig. 3D). As the terbutryne closes the acceptor side of the $\mathrm{RC}$ by replacing $\mathrm{Q}_{\mathrm{B}}$, both light-induced charges are trapped in the RC. They are removed either by slow reactions with auxiliary oxidants and reductants of unknown origin or by intraprotein charge recombination between $\mathrm{P}^{+}$and $\mathrm{Q}_{\mathrm{A}}^{-}$. The relatively fast kinetics indicates that the charge recombination should play determining role in re-opening the $\mathrm{RC}$.

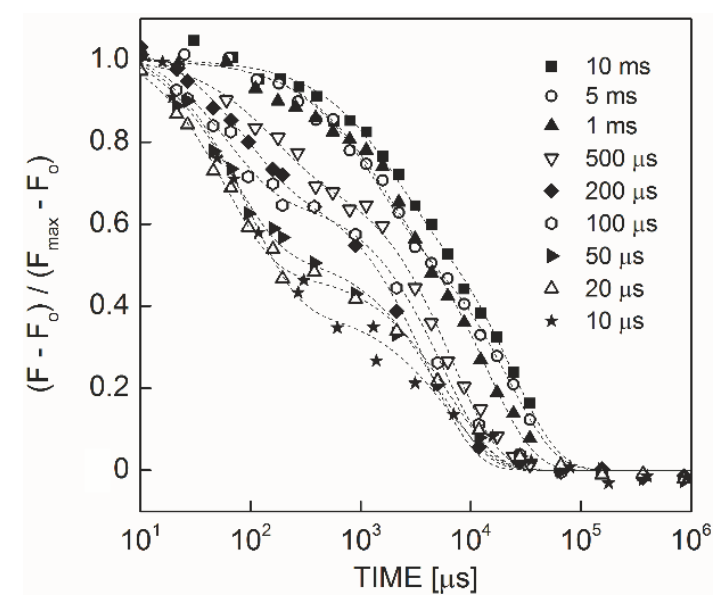

Fig. 4. Relaxation of normalized variable fluorescence generated by intensive light excitation of different durations from $10 \mu$ s to $10 \mathrm{~ms}$ in whole cells of Rhodobacter sphaeroides. 
We noted a greater decrease in the magnitude of the variable fluorescence in wild type than that in the $c y c A$ mutant (compare Figs. $3 A, C$ ). We speculate that this decrease is an indication of minor loss of the connectivity of the light-harvesting pigments to the $\mathrm{RC}$ that might include detachment of some BChls from the antenna.

Similarly, complex kinetics can be observed in the relaxation of the fluorescence if the excitation was terminated and the decay of the fluorescence state was tested by weak measuring flashes. Although the relaxation

\section{Discussion}

Phases of fluorescence induction under high light excitation: The kinetics of fluorescence induction excited by high intensity laser light is a multiphasic function of time caused by fluorescence quenchers of different origins (Fig. 2). Their kinetic overlap (appearance and disappearance) and strength determine the shape of the observed fluorescence induction. The following phases can be distinguished in dark-adapted intact cells of $R$. sphaeroides under $2 \mathrm{~W} \mathrm{~cm}^{-2}$ laser excitation at $808 \mathrm{~nm}$.

The fluorescence rises to about $90 \%$ of the maximum level within several $\mu$ s. As the rise time increases with the light intensity in linear fashion, this fast initial rise of the fluorescence is the photochemical rise and corresponds to the disappearance of the photochemical quencher $\mathrm{PQ}_{\mathrm{A}}$ via $\mathrm{PQ}_{\mathrm{A}} \rightarrow \mathrm{P}^{+} \mathrm{Q}_{\mathrm{A}}^{-}$.

The single turnover of the $\mathrm{RC}$ can produce different redox forms of the $\mathrm{RC}$ depending on the rates of rereduction of $\mathrm{P}^{+}$to $\mathrm{P}$ via bound (or unbound) cyt $c_{2}{ }^{2+}$ and re-oxidation of $\mathrm{Q}_{\mathrm{A}}^{-}$via interquinone electron transfer to $\mathrm{Q}_{\mathrm{B}}$. If both reactions are carried out, a second turnover can take place which closes the RC completely in form of $\mathrm{P}^{+}\left(\mathrm{Q}_{\mathrm{A}} \mathrm{Q}_{\mathrm{B}}\right)^{2-}$. If this happens, the fluorescence is reaching its maximum. This can occur within 100-200 $\mu$ s (practically the exchange time of cyt $c_{2}$ ) and leads to an additional rise of the fluorescence to its maximum $(100 \%)$. The lack of this component is evident in $c y c A$ mutant where the multiple turnover of the RC is inhibited.

Due to the high light intensity, the carotenoid triplet is formed in parallel with the photochemical quencher. The carotenoid triplet quencher overlaps the photochemical quencher resulting in a decrease of the maximum level of fluorescence, with an occasionally decreasing kinetic phase as observed in nitrogen atmosphere, where the triplet lifetime increases, and a disappearance of the slow phase of the photochemical rise.

As the oxygen-dependent lifetime of the carotenoid triplet is about $5 \mu \mathrm{s}$ under anaerobic conditions, fast equilibrium is established between the two quenchers that is not perturbed in the $\mu$ s time range. Later, however, continuous but slow (note the logarithmic scale in Fig. 2) decrease of the fluorescence can be observed in a very broad time scale without any noticeable and characteristic kinetic components. This is in contrast with the earlier kinetics is inherently multiphasic, the deceleration of the decay after increasing excitation flash duration from $10 \mu \mathrm{s}$ can be well recognized (Fig. 4). The demonstrative changes in the decay kinetics are terminated after sufficiently long $(>1 \mathrm{~ms})$ excitation.

The kinetics derived from induction and relaxation of the fluorescence are highly correlated in the $10^{-5}-10^{-2} \mathrm{~s}$ time range (compare Figs. 3, 4). The major changes in the kinetics observed in both cases indicate a common origin of the phenomena.

findings where six phases in the fluorescence transition kinetics of $R$. sphaeroides were reported with a series of lifetimes from $4 \mathrm{~ms}$ to $10 \mathrm{~s}$ (Bína et al. 2010). The reason for this contradiction is unclear but could be related to the different light conditions and physiological status of the bacteria or to the sudden and unexpected changes in the fluorescence transient. The decline of the fluorescence transient from the maximum to the stationary level is altered very little by the addition of uncoupler FCCP; this indicates that the membrane potential and $\mathrm{pH}$ difference makes an insignificant contribution to the changes we observed in the fluorescence induction. Additionally, the membrane potential is more likely to increase than decrease the yield of fluorescence (Bína et al. 2010). Based on our results above, we argue that the slow decline of $\mathrm{BChl}$ fluorescence after the maximum can be attributed to direct loss of BCh1* and/or to a slight increase of triplet concentration. This type of slow modulation can be achieved by molecular oxygen in the bacterial culture. The oxygen-induced deactivation of $\mathrm{BChl}$ fluorescence is diffusion controlled and proceeds via $\mathrm{S}_{1} \rightarrow \mathrm{S}_{0}$ internal conversion and $\mathrm{S}_{1} \rightarrow \mathrm{T}_{1}$ intersystem crossing both causing the decline of $\mathrm{BChl}$ fluorescence.

Determination of the rate of cyclic electron flow in intact cells: At low light intensities, a definite drop of the maximum fluorescence level can be observed in accordance with the increase of the half rise time of the fluorescence induction. This is a clear indication of the light intensity limitation of the cyclic electron transport. In this case, the rates of the cyclic electron transfer (overall rate constant $k_{\mathrm{e}}$ ) and charge losses in the RC (rate constant $k_{\text {loss, }}$ Fig. 1) can compete with that of the light-induced charge separation (rate constant $k_{\mathrm{I}}$ ). The observed fluorescence level $\mathrm{F}$ is the sum of $\mathrm{F}_{0}$ and $\mathrm{F}_{\max }$ weighted by the fractions of open $[\mathrm{PQ}]$ and closed $\left[\mathrm{P}^{+} \mathrm{Q}^{-}\right] \mathrm{RCs}$, respectively:

$$
\left.\mathrm{F}=\mathrm{F}_{0}[\mathrm{PQ}]+\mathrm{F}_{\max } \mid \mathrm{P}^{+} \mathrm{Q}^{-}\right\rfloor
$$

The kinetics of the RC states is governed by

$$
\frac{\mathrm{d}[\mathrm{PQ}]}{\mathrm{dt}}=-\mathrm{k}_{\mathrm{I}}[\mathrm{PQ}]+\left(\mathrm{k}_{\text {loss }}+\mathrm{k}_{\mathrm{e}}\right)\left[\mathrm{P}^{+} \mathrm{Q}^{-}\right]
$$


where

$$
1=[\mathrm{PQ}]+\left\lfloor\mathrm{P}^{+} \mathrm{Q}^{-}\right\rfloor
$$

Introducing the normalized variable fluorescence $\mathrm{F}_{\mathrm{v}}=$ $\left(\mathrm{F}-\mathrm{F}_{0}\right) /\left(\mathrm{F}_{\max }-\mathrm{F}_{0}\right)$, we can get

$$
\mathrm{F}_{\mathrm{v}}=\left(1-\frac{\mathrm{k}_{\mathrm{e}}+\mathrm{k}_{\text {loss }}}{\mathrm{k}_{\mathrm{I}}+\mathrm{k}_{\mathrm{e}}+\mathrm{k}_{\text {loss }}}\right)\left(1-\mathrm{e}^{-\left(\mathrm{k}_{\mathrm{I}}+\mathrm{k}_{\mathrm{e}}+\mathrm{k}_{\text {loss }}\right) \cdot \mathrm{t}}\right)
$$

Both the amplitude and the rise of the fluorescence induction curves include the rate constants. When the light intensity is high $\left(k_{\mathrm{I}}>>k_{\mathrm{e}}+k_{\text {loss }}\right)$, the RC can turn over only once in the time range of less than $1 \mathrm{~ms}$ and the photochemical quenching determines solely the kinetics of the fluorescence rise to the maximum, $\mathrm{F}_{\max }$. If we extend a time range somewhat over $1 \mathrm{~ms}$ (by using smaller light intensity), a detectable drop in the fluorescence can be observed. Due to the single turnover of the $\mathrm{RC}$, this phase represents the export of the single positive charge from $\mathrm{P}^{+}$ $\left(\mathrm{Q}_{\mathrm{B}}{ }^{-}\right.$is highly stable on the acceptor side of the $\left.\mathrm{RC}\right)$. This is actually the "loss" term in Eq. 4. If, however, the RC can perform multiple turnovers in the smaller time scale (at lower light intensity), $k_{\text {loss }}$ will be neglected and will be part of the overall rate constant of the cyclic electron transport $k_{\mathrm{e}}$. This is manifested by the gradual decrease of the stationary level (amplitude) of the fluorescence.

The two phases of the drop of the fluorescence can be analyzed based on the amplitudes of Eq. 4: the reciprocal value of $F_{v}$ should be a linear function of the reciprocal of $k_{\mathrm{I}}$. The slopes of the straight lines in double reciprocal representation give the rate constants of the thermal reactions $k_{\text {loss }}$ and $k_{\mathrm{e}}(\mathrm{Fig}$. $5 A$ ). The larger rate corresponds to the detachment of cyt $c_{2}{ }^{3+}$ from the RC (single turnover) and the smaller value to the overall cyclic rate of the electron (multiple turnover).

Another way of determination of the thermal rate constants is the fit of the set of the measured fluorescence induction curves to the exponential functions with variable amplitudes and rate constants given in Eq. 4. Using this procedure, both the steady-state fluorescence levels (amplitudes) and the rates of the fluorescence rise $\left(k_{\mathrm{I}}+k_{\mathrm{e}}\right.$ $\left.+k_{\text {loss }}\right)$ as principal information of Eq. 4 can be utilized. The solution of the problem is highly constrained as there are only two parameters to be fitted to the series of fluorescence transients at different light intensities. Using a least square method, the fit gives reasonable result that can be validated by comparison of Figs. $3 A, 5 B$.
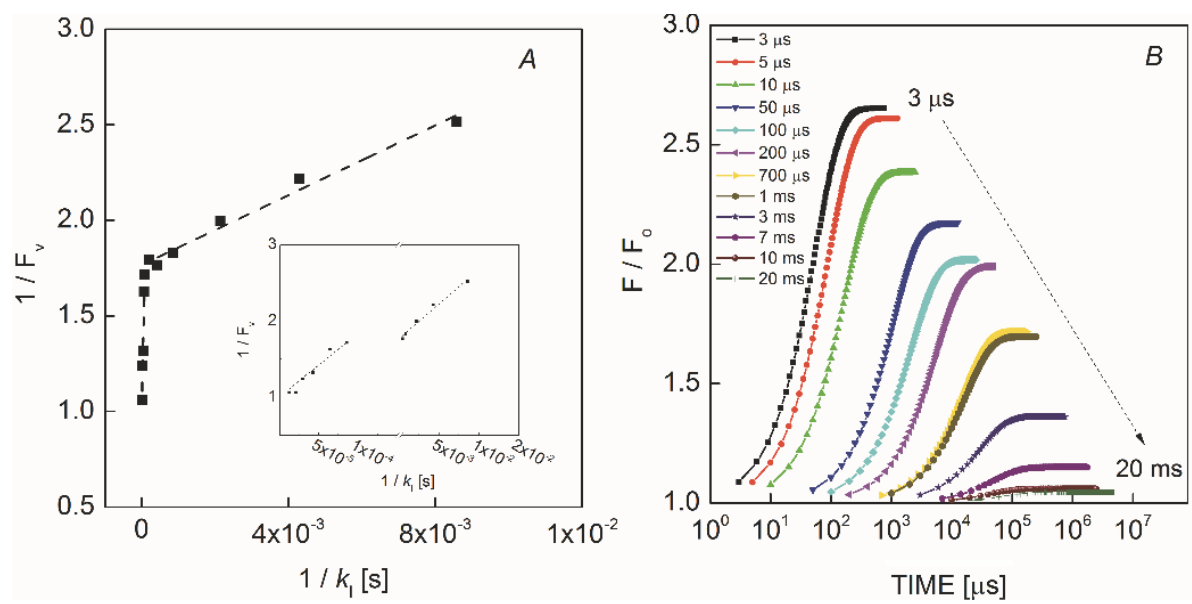

Fig. 5. Evaluation of the fluorescence induction curves of Rhodobacter sphaeroides at different excitation light intensities (Fig. $3 \mathrm{~A}$ ) according to the simplified electron transfer model in Fig. 1. $(A)$ The reaction rate constants of the thermal reactions $k$ loss (single turnover of the RC) and $k_{\mathrm{e}}$ (multiple turnover) can be derived as the slopes of the straight lines in a double reciprocal representation of $\mathrm{F}_{\mathrm{v}}$ and $k \mathrm{~s}$ (see the amplitude of Eq. 4). The inserted panel with divided 1/ke scale exposes both components. (B) Global fit of Eq. 4 to the set of fluorescence transients with parameters $k_{\text {loss }}=120 \mu \mathrm{s}^{-1}$ and $k_{\mathrm{e}}=11 \mathrm{~ms}^{-1}$ and values of duty cycle given in the box.

It is worthwhile to compare our kinetic data for whole cells with those in chromatophores obtained from other sophisticated flash assays and model calculations. The quinone reduction cycle in chromatophores was estimated to be about $1.6 \mathrm{~ms}$, including $1.2 \mathrm{~ms}$ required for the slow binding/release steps (Comayras et al. 2005). The halftime required for the released quinol to arrive and bind at the oxidizing site of the cyt $b c_{1}$ complex $\left(\mathrm{Q}_{0}\right)$ was taken as 5-10 ms (Crofts et al. 1983). The overall cycling time of electrons in chromatophore was estimated to be $13 \mathrm{~ms}$ (Geyer and Helms 2006) and 22 ms (Bína et al. 2010). However, these values are highly influenced by several factors including the arrangement of the electron transfer carriers, the redox conditions of the pools and complexes, and the ways of diffusion of the mobile species. As with the mobile cyt $c_{2}$ carriers on the donor side, the quinones in the supercomplex structure are also exposed to confinement (Comayras et al. 2005), which shortens the average distance of diffusion between the $\mathrm{Q}_{\mathrm{B}}$ and $\mathrm{Q}_{0}$ sites of the RC and cyt $b c_{1}$ complex, respectively (Joliot et al. 2005). However, it was found that the rate of diffusion was not the rate limiting step of the electron transfer (Geyer and Helms 2006). By theoretical treatment of the overall operation of the electron transfer, a model was provided 
where the significance of the reverse electron flow from the ubiquinone pool (Fig. 1) was also stressed (Klamt et al. 2008).

The fluorescence induction and relaxation kinetics offered here $120 \mu \mathrm{s}^{-1}$ rate constant for the electron exchange between $\mathrm{P}^{+}$and cyt $c_{2}{ }^{2+}$ as the major pathway of charge export on the donor side of the intact cell of $R$. sphaeroides. This process offers the dominating component in single turnover of the RC. In multiple turnover, however, when the cyclic (from RC to RC) electron transport is operating, a rate constant of $11 \mathrm{~ms}^{-1}$ was found which falls in the wide range of values discussed above. The cyclic electron transfer includes several steps and the slowest one determines the overall measured rate. The bottle neck can be the binding/

\section{References}

Asztalos E., Italiano F., Milano F. et al.: Early detection of mercury contamination by fluorescence induction of photosynthetic bacteria. - Photoch. Photobio. Sci. 9: 1218-1223, 2010.

Asztalos E., Sipka G., Maróti P.: Fluorescence relaxation in intact cells of photosynthetic bacteria: donor and acceptor side limitations of reopening of the reaction center. - Photosynth. Res. 124: 31-44, 2015.

Bína D., Litvín R., Vácha F.: Absorbance changes accompanying the fast fluorescence induction in the purple bacterium Rhodobacter sphaeroides. - Photosynth. Res. 105: 115-121, 2010.

Chi S.C., Mothersole D.J., Dilbeck P. et al.: Assembly of functional photosystem complexes in Rhodobacter sphaeroides incorporating carotenoids from the spirilloxanthin pathway. - Biochim. Biophys. Acta 1847: 189-201, 2015.

Comayras F., Jungas C., Lavergne J.: Functional consequences of the organization of the photosynthetic apparatus in Rhodobacter sphaeroides. I. Quinone domains and excitation transfer in chromatophores and reaction center antenna complexes. - J. Biol. Chem. 280: 11203-11213, 2005.

Crofts A.R., Meinhardt S.W., Jones K.R., Snozzi M.: The role of the quinone pool in the cyclic electron-transfer chain of Rhodopseudomonas sphaeroides: A modified Q-cycle mechanism. - Biochim. Biophys. Acta 723: 202-218, 1983.

de Rivoyre M., Ginet N., Bouyer P., Lavergne J.: Excitation transfer connectivity in different purple bacteria: a theoretical and experimental study. - Biochim. Biophys. Acta 1797: 17801794, 2010.

Donohue T.J., Kaplan S.: Genetic techniques in rhodospirillaceae. - Methods Enzymol. 204: 459-485, 1991.

Duysens L.N.M.: Transfer of light energy within the pigment systems present in photosynthesizing cells. - Nature 168: 548550,1951

Geyer T., Helms V.: A spatial model of the chromatophore vesicles of Rhodobacter sphaeroides and the position of the cytochrome $b c_{1}$ complex. - Biophys. J. 91: 921-926, 2006.

Joliot P., Joliot A., Vermeglio A.: Fast oxidation of the primary electron acceptor under anaerobic conditions requires the organization of the photosynthetic chain of Rhodobacter sphaeroides in supercomplexes. - BBA-Bioenergetics 1706: 204-214, 2005.

Kautsky H., Hirsch A.: [New experiments on carbonic acid assimilation.] - Naturwissenschaften 19: 964, 1931. [In German] unbinding of mobile quinone and cytochrome $c_{2}$ species to the RC (and to the cyt $b c_{1}$ complex) and/or their pathway through the corresponding pools. The effects of the redox state of the pools, intactness of the macromolecular complexes, and the crowding of the molecular constituents of the apparatus can have major influence on the observed kinetics as was demonstrated under different treatments of the cells. As these parameters are determined by the physiological status of the bacteria, the fluorescence kinetic traces can be used to monitor the internal electron transfer processes and the photosynthetic capacity if the proper protocol and evaluation method are available. The present work served this purpose together with congratulations to Govindjee for his birthday. We wish him many happy returns of the day.

Kis M., Asztalos E., Sipka G., Maróti P.: Assembly of photosynthetic apparatus in Rhodobacter sphaeroides as revealed by functional assessments at different growth phases and in synchronized and greening cells. - Photosynth. Res. 122: 261273, 2014.

Kis M., Sipka G., Asztalos E. et al.: Purple non-sulfur photosynthetic bacteria monitor environmental stresses. - J. Photoch. Photobio. B 151: 110-117, 2015.

Klamt S., Grammel H., Straube R. et al: Modelling the electron transport chain of purple nonsulfur bacteria. - Mol. Syst. Biol. 4: 156, 2008.

Koblizek M., Shih J.D., Breitbart S.I. et al.: Sequential assembly of photosynthetic units in Rhodobacter sphaeroides as revealed by fast repetition rate analysis of variable bacteriochlorophyll a fluorescence. - Biochim. Biophys. Acta 1706: 220-231, 2005.

Kocsis P., Asztalos E., Gingl Z., Maróti P.: Kinetic bacteriochlorophyll fluorometer. - Photosynth. Res. 105: 73-82, 2010.

Maróti P.: Kinetics and yields of bacteriochlorophyll fluorescence: redox and conformation changes in reaction center of Rhodobacter sphaeroides. - Eur. Biophys. J. 37: 1175-1184, 2008.

Maróti P.: Induction and relaxation of bacteriochlorophyll fluorescence in photosynthetic bacteria. - In: Pessarakli M. (ed): Handbook of Photosynthesis, $3^{\text {rd }}$ ed. Pp. 463-483. CRC Press, Boca Raton - London - New York 2016.

Niederman R.A.: Development and dynamics of the photosynthetic apparatus in purple phototrophic bacteria. - Biochim. Biophys. Acta 1857: 232-246, 2016.

Sambrook J., Fritsch E.F., Maniatis T.: Molecular cloning: a laboratory manual, $2^{\text {nd }}$ ed. Page A.1. Cold Spring Harbor Laboratory Press, New York 1989.

Siström W.R.: The kinetics of the synthesis of photopigments in Rhodopseudomonas spheroides. - J. Gen. Microbiol. 28: 607616, 1962.

Siström, W.R., Transfer of chromosomal genes mediated by plasmid r68.45 in Rhodopseudomonas sphaeroides. - J. Bacteriol. 131: 526-532, 1977

Stirbet A., Govindjee: Chlorophyll $a$ fluorescence induction: a personal perspective of the thermal phase, the J-I-P rise. Photosynth. Res. 113: 15-61, 2012.

Trissl H.W.: Antenna organization in purple bacteria investigated by means of fluorescence induction curves. - Photosynth. Res. 47: $175-185,1996$. 